\title{
Efecto de la aplicación de enmiendas orgánicas, como complemento a la fertilización química en la producción de pimiento (Capsicum annum L.), en la zona de Babahoyo
}

\author{
Ing. Agrop. Victor Molina Barbotó, \\ Ing. Agro. Joffre León Paredes, \\ Ing. Agr. Ricardo Chávez Betancourt, \\ Ing. Agr. Darío Dueñas Alvarado, \\ Ing. Agrop. Eduardo Yance Jiménez, \\ Ing. David Mayorga Arias, \\ Arq. Pedro Rodríguez Vargas, \\ Mvz. William Filian Hurtado, \\ Juan Salina Ochoa, \\ Ing. Cristina Maldonado Campozano, \\ Universidad Técnica de Babahoyo, Los Ríos-Ecuador
}

\begin{abstract}
Pepper (Capsicum annum 1.) belongs to the genus Capsicum of the Solanaceae families. Its fruit is green. It has a high content of vitamins A and C. This fruit, however, is of great importance to the human population. The stud of pepper (Capsicum annuum 1.) was carried out in the fields located in the Technical University of Babahoyo, Faculty of Agricultural Sciences in Los Ríos province, Ecuador. This work was carried out between the months of September to January 2016. The planting was done with the hybrid material, Salvador, in plots of $11.9 \mathrm{~m} 2$. The statistical design used was completely randomized blocks with 10 treatments and 4 replicates. The useful area of the plot was $476 \mathrm{~m} 2$. At the end of the crop cycle, the following was obtained: plant height, days to flowering, days to harvest, number of fruits per plant, fruit length, weight of fruit, fruit diameter, size of fruit, days to maturity, yield, and cost-benefit ratio. Based on the results of the present study, it was demonstrated that the application of organic matter in combination of a fertilization program affected the agronomic performance of the cultivation of peppers under the field conditions in which it was developed. Based on statistical analysis, we can also mention that
\end{abstract}


Salvador hybrid material used in this trial presents an extension in the days corresponding to the flowering and ripening under the applications of functional organic material. This factor coupled with the application of chemical fertilizer affects performance. The best agronomic performance of the fruit was found in treatment, Bokashi $4000 \mathrm{Kg} / \mathrm{ha}$, which presented the best features based on the number of fruits, fruit diameter, and length off. As a result of the application of fertilizers and amendments, it was found that they had influence on the plant height measurements made in the evaluated treatments.

Keywords: Hybrid, fertilization, amendments, treatments and fruits.

\section{Resumen}

El pimiento (Capsicum annum L.) pertenece al género Capsicum de las familias de las Solanáceas, sus frutos se los consume en verde. Tiene un alto contenido de vitaminas A Y C; lo cual se constituye en un alimento de mucha importancia para la población humana. El trabajo se realizó en los campos, ubicados en la Universidad Técnica de Babahoyo, Facultad de Ciencias Agropecuarias en la provincia de los Ríos, el presente trabajo se realizó entre los meses de septiembre-enero del 2016. La siembra se realizó con el material híbrido Salvador, en parcelas de $11.9 \mathrm{~m} 2$. El diseño estadístico utilizado fue bloques completamente al azar con 10 tratamientos y 4 repeticiones, el área útil de la parcela fue $476 \mathrm{~m} 2$. Al final del ciclo del cultivo se evaluó: altura de planta, días a la floración, días a la cosecha, número de frutos por planta, longitud de fruto, peso de fruto, diámetro de fruto, tamaño de fruto, días a la maduración, rendimiento, relación beneficiocosto. Basados en los resultados del presente ensayo se demostró, que la aplicación de materia orgánica en combinación de un programa de fertilización, incidieron en el comportamiento agronómico del cultivo de pimiento, bajo las condiciones de campo en que se desarrolló el mismo. Como consecuencia de las aplicaciones de fertilizantes y enmiendas realizadas, se encontró que las mismas tuvieron influencia sobre la altura de la planta en las mediciones realizadas en los tratamientos evaluados. Realizados los análisis de estadística también se puede mencionar que el material híbrido Salvador utilizado en este ensayo, presenta una prolongación en los días correspondientes a la floración y maduración, bajo las aplicaciones de materia orgánica funcional, factor que sumado a la aplicación de fertilización química incide en el rendimiento. El mejor comportamiento agronómico del fruto se encontró en el tratamiento Bocashi $4000 \mathrm{Kg} / \mathrm{ha}$, el cual presentó mejores características en número de frutos, diámetro de frutos y longitud de frutos; siendo este estadísticamente igual a la dosis de Bocashi $3500 \mathrm{Kg} / \mathrm{ha}$. Los rendimientos presentados sobrepasaron 
el promedio obtenido a nivel nacional, bajo las condiciones del ensayo. El mayor efecto se presentó en el tratamiento Algasoil $200 \mathrm{Kg} /$ ha que están por encima del testigo.

Palabras claves: Híbrido, fertilización, enmiendas, tratamientos, frutos.

\section{Introduction}

El pimiento (Capsicum annum L.) pertenece al género Capsicum de las familias de las Solanáceas, sus frutos se los consume en verde. Tiene un alto contenido de vitamienas A Y C; lo cual se constituye en un alimento de mucha importancia para la población humana.

Muchas son las variedades de pimiento que se siembran en nuestro medio, en algunos casos se ha tenido éxito en otros no, debido a que su explotación no ha sido manejada de forma adecuada; por lo que no se ha logrado el potencial de rendimiento, además también inciden el uso de variedades no adaptadas a nuestras condiciones o densidades de siembra no acordes al genotipo, tipo de suelo, clima, riego y nutrición entre otras causas etc.

En el Ecuador, se siembran alrededor de 1145 hectáreas con un rendimiento promedio de 4,58 ton/ha. Este promedio resulta bajo en comparación a los registrados por otros países, lo cual se debe a varios factores, entre ellos los señalados en líneas anteriores.

Las enmiendas orgánicas son productos elaborados a base de residuos $\mathrm{y}$ desechos vegetales elaborados artesanalmente. Estas enmiendas se encuentran de forma natural en el suelo, habiendo sido afectadas por el uso excesivo de fertilizantes químicos, labores de suelo u otros agroquímicos, que degradan sus componentes y disminuye su cantidad en el suelo.la utilización de enmiendas es una tecnología utilizada desde inicio de la explotación de cultivos y que durante los últimos años se vuelve de actualidad sin embargo, no se encuentra debidamente estudiado y que presenta muchas perspectivas a futuro a la creciente demanda de productos sanos.

Mediante el empleo de abonos orgánicos es posible mantener e incrementar el rendimiento de frutos, mejorando su calidad. También, es necesario para su aplicación determinar la época más apropiada en que se debe realizarse el trasplante, es decir, conocer la edad apropiada en que las plantas deben ser llevadas del semillero al campo definitivo para que las enmiendas surtan su efecto positivo.

Los aportes que da la materia orgánica ha logrado en aumentar los niveles de vida microbiológica del suelo también ha contribuido a mejorar productos alternativos que garanticen una producción sustentable y amigable con el ambiente. 


\section{OBJETIVOS}

\section{General}

"Efecto de la aplicación de enmiendas orgánicas, como complemento a la fertilización química en la producción de pimiento"

\section{Específicos}

- Determinar el comportamiento agronómico del cultivo de pimiento a la aplicación de las enmiendas orgánicas.

- Evaluar las dosis de las enmiendas que generen mayor rendimiento en el cultivo de pimiento.

\section{REVISIÓN DE LITERATURA}

Según Espinoza (2007), la fertilización del cultivo se debe en base al análisis químico del suelo, pero de forma general se recomienda por hectáreas aplicar al suelo $140 \mathrm{Kg}$ de nitrógeno, 65 de fósforo, 1190 de potasio, 25 de magnesio y 30 de azufre; para un rendimiento de $5 \mathrm{t} / \mathrm{ha}$.

El CIAT (2003), nos indica que solo con aplicaciones de estiércol de gallinas con 8 toneladas por hectárea, $60 \mathrm{Kg}$ de nitrógeno y $40 \mathrm{Kg}$ de fósforo, antes del trasplante da un mejor resultado.

Según www.ecuaquímica.com (2016), el agasoil es un fertilizante orgánico de suelos, fabricado con algas marinas, pastes de soya, sangre mineralizada y aditivo algínico en forma de coloides producidos por algas marinas. Debido a su contenido natural de minerales y aminoácidos, forman un balance perfecto y completo de los elementos esenciales del suelo.

\begin{tabular}{|c|c|}
\hline \multicolumn{2}{|c|}{ Composición química del Algasoil } \\
\hline Nitrógeno total & $2,00 \%$ \\
\hline Fósforo (Fosfato-P2O5) & $2,00 \%$ \\
\hline Potasio (K2O) & $2,00 \%$ \\
\hline Materia Orgánica & $70,00 \%$ \\
\hline Aditivos de tipo Alginato & $10,00 \%$ \\
\hline Gránulos (DE 1-4,7 MM) & mayor $=8 \%$ \\
\hline $\begin{array}{c}\text { Promedios de compactación De } \\
\text { Gránulos N }\end{array}$ \\
mayor $=6 \%$ \\
\hline Color y apariencia & $\begin{array}{c}\text { Gránulos } \\
\text { Cafés }\end{array}$ \\
\hline
\end{tabular}

En cuanto al humus según (Colacelli, 1997; Artículo de revista) indica que producto también conocido como vermicompost o lombricompuesto, es el resultado de la transformación de materiales orgánicos por las lombrices. Existen varias especies, la más utilizada es la llamada lombriz roja californiana (Eisenia foetida). El proceso consiste en el pasaje por el intestino de la lombriz de materiales orgánicos que se mezclan en el tracto 
digestivo con elementos minerales, microor-ganismos $\mathrm{y}$ fermentos, que producen cambios bioquímicos en el sustrato.

Las deyecciones de las lombrices quedan así enriquecidas y predigeridas lo cual acelera el proceso de mineralización y humificación de la materia orgánica que lo compone. Una de las alternativas es posibilitar el ciclo fermentativo, sobre estiércol vacuno por ejemplo, procediendo luego al sembrado de las lombrices, con lo cual se obtiene un producto de inmejorable calidad.

Este humus posee un $\mathrm{pH}$ 6,8 a 7,2 o sea neutro, aportando además sustancias estimuladoras del crecimiento vegetal (auxinas, giberelinas, citoquininas, etc.) y una población bacteriana benéfica. En la siguiente tabla se comparan los contenidos de algunos elementos con el lombricompuesto. Del análisis de estos valores se desprende que el lombricompuesto posee entre 3 a 4 veces más cantidad de elementos que los otros estiércoles.

\begin{tabular}{|l|c|c|c|c|c|}
\hline Tipo de estiércol & $\begin{array}{c}\text { Materia } \\
\text { Seca }\end{array}$ & $\mathbf{N}$ & P2O5 & OK2 & OCa \\
\hline Equino & 33 & 0,67 & 0,25 & 0,55 & 0,2 \\
\hline Bovino & 18 & 0,6 & 0,15 & 0,45 & 0,15 \\
\hline Gallina & 45 & 1 & 0,8 & 0,4 & 0 \\
\hline Lombricompuesto & $30-50$ & 2,42 & 3,74 & 1,1 & 2,47 \\
\hline
\end{tabular}

Si midiéramos con algún parámetro la fertilidad "incorporada" al suelo por el lombricompuesto veríamos que es mayor en 4-5 veces respecto a la incorporación que hacen otro tipo de estiércoles.

Si por alguna eventualidad se aplicaron cantidades excesivas, esto no causará problemas ya que la liberación de los principios activos se dosifican en forma adecuada. Otro aspecto a destacar es que a diferencia de otros estiércoles, el aporte de bacterias benéficas es de varios billones/g mientras que en los estiércoles comunes no pasan de pocos millones/g.

Esta incorporación de vida al suelo permite la liberación de compuestos no asimilables por los vegetales, poniéndolos a disposición de los mismos, esto es particularmente cierto en el caso del fósforo.

Si comparamos el contenido de nutrientes de los primeros $15 \mathrm{~cm}$ de suelo con los excrementos de las lombrices que lo habitan obtenemos los siguientes resultados:

5 veces más ricos en nitrógeno nítrico.

2 veces más ricos en calcio asimilable.

2,5 veces más ricos en magnesio asimilable.

7 veces más ricos en fósforo asimilable.

11 veces más ricos en potasio asimilable. 
Suquilanda (2014), manifiesta que el Bocashi que resulta de la fermentación de desechos de carácter vegetal y animal al que se le puede agregar elementos de origen mineral para enriquecerlo (cal, roca fosfórica) y microorganismos para activar el proceso fermentativo. Este abono es muy seguro y eficiente, ya que contiene los elementos necesarios para la nutrición de las plantas y posee una alta carga de microorganismos benéficos.

\begin{tabular}{|c|c|c|}
\hline \multicolumn{3}{|c|}{ COMPOSICIÓN OUÍMICA DEL BOCASHI } \\
\hline Materia Orgánica & $22.30 \%$ & Alto \\
\hline Nitrógeno Total & $1.12 \%$ & Alto \\
\hline P ppm & $2.17 \%$ & Alto \\
\hline K (meq/100) & $1.72 \%$ & Alto \\
\hline Ca (meg/100) & $6.23 \%$ & Alto \\
\hline Aluminio (Al) & $3.00 \%$ & Bajo \\
\hline Magnesio (meq/100g) & 3.09 & Bajo \\
\hline Boro ppm & 5.83 & Bajo \\
\hline Hierro (Fe) (ppm) & $0.5 \%$ & Bajo \\
\hline Cobre (Cu) (ppm) & 0.01 & Bajo \\
\hline Azufre (S) 8ppm & 3.00 & Bajo \\
\hline
\end{tabular}

Los sustratos utilizados en la producción de plantines son muy diversos, variando en su complejidad desde turba de Sphagnum pura, o en mezclas de proporciones volumétricas variables con perlita, arenas, micas, materiales compostados o suelo extraído de capas u horizontes superficiales. Estas mezclas son preparadas por los propios productores o por firmas comerciales.

Normalmente se presentan estériles y con sus atributos fisicoquímicos como $\mathrm{pH}$, conductividad electrolítica, densidad aparente, humedad, relación $\mathrm{C} / \mathrm{N}$ y capacidad de intercambio iónico, ajustados $\mathrm{y}$ especificados. Los plantines presentan una elevada demanda de nutrientes minerales, en parte como resultado de su alta tasa de crecimiento en relación con las plantas adultas.

Además de representar una fuente de nutrientes, los sustratos a utilizar en la producción de plantines deben poseer buena retención y disponibilidad de agua, promover un eficiente intercambio de gases y dar soporte a la planta (Javier de Grazia, 2006; Artículo de revista).

Según MUNDO VERDE (2015), El fossil es un fertilizante micro pulverizado, $100 \%$ natural para toda clase de cultivos, contiene fósiles de micro algas de aguas dulces con un alto nivel de pureza. Posee Sílice amorfa y más de 19 minerales y micro elementos muy importantes y básicos en el desarrollo nutricional de las plantas. 
Composición química del Fossil

\begin{tabular}{|l|c|}
\hline Ingrediente activo & Microalgas fosilizadas \\
\hline Elementos & \\
\hline Aluminio $(\mathrm{A})$ & $\mathbf{3 , 6 5 \%}$ \\
\hline Boro $(\mathrm{B})$ & $\mathbf{0 , 1 6 \%}$ \\
\hline Calcio $(\mathrm{Ca})$ & $\mathbf{1 , 1 0 \%}$ \\
\hline Hierro $(\mathrm{Fe})$ & $\mathbf{2 , 7 0 \%}$ \\
\hline Silice $\left(\right.$ Como $\left.\mathrm{SiO}_{2}\right)$ & $\mathbf{8 6 , 4 0 \%}$ \\
\hline
\end{tabular}

\section{MATERIALES Y MÉTODOS}

\section{Ubicación y descripción del sitio experimental}

El presente trabajo de investigación se realizó en los terrenos de la granja experimental de la Facultad de Ciencias Agropecuarias de la Universidad Técnica de Babahoyo, ubicada en el Kilómetro 7 1/2 de la vía a Montalvo.

La zona presenta un clima tropical húmedo según clasificación de HOLDRIBGE, con temperatura anual promedio de 25,7 0C, una precipitación de $2791.4 \mathrm{~mm} / \mathrm{año}$, humedad relativa de $76 \%$ y 804.7 horas de heliofanía. Coordenadas geográficas de longitud Oeste 790 32', latitud sur 010 47'49", altitud $7 \mathrm{msnm}^{1}$.

\section{Material Vegetativo}

Se utilizará como material de siembra el híbrido Salvador:

Ciclo: 85 días inicio de cosecha

Forma del fruto: Alargado

Color del fruto: Verde oscuro

Paredes del fruto: Gruesas de 3,5 mm

Dimenciones del fruto: $17 \mathrm{~cm}$. de largo x $5 \mathrm{~cm}$. de diámetro

Hábito de crecimiento: Semi-indeterminado

Población/ha: 28,000 a 33,000 plantas

Producción aproximada: $30,000 \mathrm{Kg}$.

\section{Factores Estudiados}

A: Dosis de aplicación de enmiendas

B: Comportamiento agronómico del cultivo del pimiento

\section{Diseño Experimental}

El diseño que se utilizó fue bloques completamente al Azar con 10 tratamientos y 4 repeticiones. 


\section{Tratamientos}

Se utilizó los siguientes tratamientos:

\begin{tabular}{|c|c|c|c|c|c|}
\cline { 2 - 6 } \multicolumn{1}{c|}{} & $\begin{array}{c}\text { Fertilización } \\
\text { Química }\end{array}$ & Humus & Bocashi & Fossil & Algasoil \\
\cline { 2 - 6 } \multicolumn{1}{c|}{} & & & $\mathrm{Kg} / \mathrm{ha}$ & & \\
\hline 1 & $\mathrm{FQ} 1$ & 3000 & & & \\
\hline 2 & & 4000 & & & \\
\hline 3 & FQ 1 & $\mathrm{XXXX}$ & 4000 & & \\
\hline 4 & & $\mathrm{XXXX}$ & 3500 & & \\
\hline 5 & $\mathrm{FQ} 1$ & $\mathrm{XXXX}$ & $\mathrm{XXXX}$ & 100 & \\
\hline 6 & & $\mathrm{XXXX}$ & $\mathrm{XXXX}$ & 150 & \\
\hline 7 & FQ 1 & $\mathrm{XXXX}$ & $\mathrm{XXXX}$ & $\mathrm{XXXX}$ & 200 \\
\hline 8 & & $\mathrm{XXXX}$ & $\mathrm{XXXX}$ & $\mathrm{XXXX}$ & 400 \\
\hline 9 & FQ 1 & $\mathrm{XXXX}$ & $\mathrm{XXXX}$ & $\mathrm{XXXX}$ & $\mathrm{XXXX}$ \\
\hline 10 & FQ 1 & $\mathrm{XXXX}$ & $\mathrm{XXXX}$ & $\mathrm{XXXX}$ & $\mathrm{XXXX}$ \\
\hline 160
\end{tabular}

FQ1: 160-60-130-50; N,P2O5,K2O,S,ZnO Kg/ha ( recomendaciones del INIAP de nutrientes edáficos para pimiento)

FQ2: 99-18-54; N,P2O5,K2O Kg/ha (testigo convencional)

Las aplicaciones se realizaron semanalmente a partir del trasplante.

\section{Dimensiones del lote experimental}

Tratamientos: 10

Repeticiones: 4

Total de parcelas: 40

Longitud de la unidad experimental: $3.4 \mathrm{~m}$

Ancho de la unidad experimental: $3.5 \mathrm{~m}$

Área de la unidad experimental: $11.9 \mathrm{~m} 2$

Longitud de la parcela: $30 \mathrm{~m}$

Ancho de la parcela: $17 \mathrm{~m}$

Área de la parcela: $731 \mathrm{~m} 2$

Área útil de la parcela: $476 \mathrm{~m} 2$

\section{Manejo del ensayo}

\section{Semillero}

Se realizó el semillero en vasos descartables, a los cuales se les rellenó con sustrato de suelo, previamente desinfectado. Para desinfectarlo se realizó la preparación de suelo con azadón la cantidad necesaria. Posteriormente se aplicó agua caliente al sustrato, se añadió ceniza vegetal $50 \mathrm{Kg} / \mathrm{m} 2$ y cal $0.15 \mathrm{Kg} / \mathrm{m} 2$. Adicionalmente se aplicó cepas de Trichoderma harzianum, para el control de hongos de suelo, y bocashi como fertilizante. 


\section{Preparación del suelo}

Se realizó la limpieza del terreno con un pase de rom plow y uno de rastra, posteriormente se hizo la aplicación de 1,2 1/ha de paraquat. Se realizó un análisis de suelo después del laboreo del mismo.

\section{Trasplante}

Se realizó a los 21 días después de la siembra del semillero, se utilizó las plantas más sanas posibles. En el hoyo de siembra se aplicó una disolución de cepas de Trichoderma harzianum a razón de $250 \mathrm{~g} / \mathrm{ha}$. El distanciamiento que se utilizó fue de $0,6 \mathrm{~m}$ entre planta y $1 \mathrm{~m}$ entre hileras.

\section{Fertilización}

La fertilización se aplicó de manera semanal después del trasplante de las plántulas a campo aprovechando las horas de la mañana para evitar estrés de las mismas y previo a un riego. Esta labor se realizó planta por planta, realizando tres hoyos a manera de trípode en donde se depositó y se tapó. La aplicación de fertilizantes se realizó en mezcla de todos los productos para aprovechar las aplicaciones, se pesó la dosis recomendadas en una balanza para lograr exactitud y confiabilidad en las mismas.

El testigo se manejó con las actividades que realiza el agricultor comúnmente, se aplicó 4 sacos de urea, 2 sacos de fertilizantes completo 8 $20-20$ y 1 saco de muriato de potasio (100 Kg de N, $20 \mathrm{Kg}$ de fósforo y 50 $\mathrm{Kg}$ de potasio); distribuido: al trasplante todo el completo, a los 20 días del mismo ( $50 \%$ dosis de $\mathrm{N}$ y K), 35 días (50\% dosis de $\mathrm{K})$ y 50 días (50\% dosis de N), después de la siembra. Adicionalmente se aplicó Evergreen 11/ha como alternativa.

\section{Riego}

Se realizó el riego al trasplante dirigido al hoyo y posteriormente cada 7 días para aprovechar la descomposición de la materia orgánica aplicada, evitando encharcamientos, mediante el uso de mangueras.

\section{Manejo de malezas}

Para el manejo de malezas gramíneas y hoja ancha se utilizó control cultural manual con rabón, las veces que fue necesario.

\section{Manejo de plagas y enfermedades}

En esta labor se utilizó las recomendaciones facilitadas por el INIAP (Instituto Nacional Autónomo de Investigaciones Agropecuarias) para umbrales económicos de insecto. Para el control de masticadores de hojas se utilizó extracto de neen en dosis de $1 \mathrm{~L} /$ ha y para el control de Spodoptera y masticadores de fruto se aplicó extracto de ají en dosis de 1,5L/ha. 
Para el control de enfermedades de suelo se utilizó sulfato de cobre en dosis de $0,8 \mathrm{~L} / \mathrm{ha}$ cada mes y para el control de enfermedades foliares Fosetil-A en dosis de $200 \mathrm{gr} / \mathrm{ha}$ cada 15 días.

\section{Datos evaluados}

\section{Altura de planta 30 y 60 días después de la siembra}

Se evaluó en 10 plantas al azar por tratamiento, tomando el valor desde el nivel del suelo hasta el último brote apical desarrollado; en las fechas señaladas. Se expresó en centímetros.

\section{Días a la floración}

Se midió en 10 plantas al azar por tratamiento, cuando el cultivo presentó el 50\% de plantas con flor abierta

\section{Días a la cosecha}

Se tomó en las mismas 10 plantas donde se efectuó el registro anterior, y se evaluó, desde la siembra en el semillero.

\section{Número de frutos por planta}

Se evaluó en 10 plantas al azar por tratamiento, contando desde el primer fruto recogido hasta el último que se pueda alcanzando a cosechar.

\section{Tamaño de fruto (longitud)}

Se tomó en 10 frutos al azar por tratamiento, y se midió desde el pedúnculo hasta la punta del mismo y se expresó en centímetros.

\section{Peso de fruto}

Se evaluó en 10 plantas al azar por tratamiento, se expresó en gramos.

\section{Diámetro de fruto}

En los frutos en que se determinó la longitud, se midió el diámetro de la parte media del mismo, utilizando un calibrador.

\section{Días a maduración}

Se evaluó en 10 plantas al azar por tratamiento, y se consideró el lapso desde la siembra en el semillero hasta cuando alcanzó el 75\% de frutos pintones (colores rojo y verde al mismo tiempo). 


\section{Rendimiento}

Se evaluó en 10 plantas seleccionadas contando todos los frutos recogidos, se utilizó una balanza de precisión para su peso y se expresó en gramos, posteriormente se llevó a $\mathrm{Kg} / \mathrm{ha}$.

\section{RESULTADOS}

\section{Altura de planta}

En el Cuadro 1, se observan los promedios de altura de plantas evaluadas a los 30 y 60 días después de la siembra. Los valores alcanzaron alta significancia estadística al $5 \%$ de probabilidades.

En la evaluación de altura de planta realizada a los 30 días después de la siembra, se encontró que el tratamiento Humus $4000 \mathrm{Kg} / \mathrm{ha}(30.75 \mathrm{~cm})$, fue estadísticamente igual a los tratamientos: Humus $3000 \mathrm{Kg} / \mathrm{ha}(27.2 \mathrm{~cm})$, Bocashi $4000 \mathrm{Kg} / \mathrm{ha}(28.05 \mathrm{~cm})$, Fossil $150 \mathrm{Kg} / \mathrm{ha}(28.1 \mathrm{~cm})$ y Algasoil 200 $\mathrm{Kg} / \mathrm{ha}(29.5 \mathrm{~cm})$; que a su vez fueron estadísticamente iguales entre sí. Sin embargo fueron estadísticamente superiores a los tratamientos Bocashi 3500 $\mathrm{Kg} / \mathrm{ha}(26.0 \mathrm{~cm})$, Fossil $100 \mathrm{Kg} / \mathrm{ha}(26.65 \mathrm{~cm})$, Algasoil $400 \mathrm{Kg} / \mathrm{ha}(25.9$ $\mathrm{cm}), \mathrm{FQ}(6.75 \mathrm{~cm})$ y testigo $(25.75 \mathrm{~cm})$. el coeficiente de variación fue de $9.80 \%$.

En la evaluación de altura de planta realizada a los 60 días, se determinó que el tratamiento Bocashi $3500 \mathrm{Kg} / \mathrm{ha}$ fue estadísticamente igual a los tratamientos: Humus $4000 \mathrm{Kg} / \mathrm{ha}(50.8 \mathrm{~cm})$, Fossil $150 \mathrm{Kg} / \mathrm{ha}(48.35$ $\mathrm{cm})$, Algasoil $200 \mathrm{Kg} / \mathrm{ha}(51.2 \mathrm{~cm})$ y FQ. $(50.2 \mathrm{~cm})$. Pero fueron superiores estadísticamente a los tratamientos: Humus $3000 \mathrm{Kg} / \mathrm{ha}(45.85 \mathrm{~cm})$, Bocashi $4000 \mathrm{Kg} / \mathrm{ha}(45.6 \mathrm{~cm})$, Fassil $100 \mathrm{Kg} / \mathrm{ha}(43.85 \mathrm{~cm})$, Algasoil $(44.7 \mathrm{~cm}) \mathrm{y}$ testigo $(44.4 \mathrm{~cm})$. El coeficiente de variación fue de $11.83 \%$.

Cuadro 1. Promedio de altura de planta a los 30 y 60 días después de la siembra de pimiento, con la aplicación de enmiendas orgánicas, como complemento a la fertilización química en la zona de Babahoyo.

\begin{tabular}{|c|c|c|}
\hline \multirow{2}{*}{ Tratamientos (*) } & \multicolumn{2}{|c|}{ Altura de planta $(\mathbf{c m})$} \\
\cline { 2 - 3 } & 30 d.d.s. & $\mathbf{6 0}$ d.d.s. \\
\hline FQ + Humus $3000 \mathrm{Kg} / \mathrm{ha}$ & $27,20 \mathrm{ab}$ & $45,85 \mathrm{~b}$ \\
\hline FQ + Humus $4000 \mathrm{Kg} / \mathrm{ha}$ & $30,75 \mathrm{a}$ & $50,80 \mathrm{ab}$ \\
\hline FQ + Bocashi $4000 \mathrm{Kg} / \mathrm{ha}$ & $26,00 \mathrm{~b}$ & $56,40 \mathrm{a}$ \\
\hline FQ + Bocashi $3500 \mathrm{Kg} / \mathrm{ha}$ & $28,05 \mathrm{ab}$ & $45,60 \mathrm{~b}$ \\
\hline FQ + Fossil $100 \mathrm{Kg} / \mathrm{ha}$ & $25,65 \mathrm{~b}$ & $43,85 \mathrm{~b}$ \\
\hline FQ + Fossil $150 \mathrm{Kg} / \mathrm{ha}$ & $28,10 \mathrm{ab}$ & $48,35 \mathrm{ab}$ \\
\hline FQ + Algasoil $200 \mathrm{Kg} / \mathrm{ha}$ & $29,50 \mathrm{ab}$ & $51,20 \mathrm{ab}$ \\
\hline FQ + Algasoil 400 Kg/ha & $25,90 \mathrm{~b}$ & $44,70 \mathrm{~b}$ \\
\hline FQ. 160-60-130-50-5 & $26,75 \mathrm{~b}$ & $50,20 \mathrm{ab}$ \\
\hline Testigo convencional & 25,75 & 44,4 \\
$90-18-54$ & & \\
\hline
\end{tabular}




\begin{tabular}{|c|c|c|}
\hline Promedios & $\mathbf{2 7 , 3 7}$ & $\mathbf{4 8 , 1 4}$ \\
\hline Significacncia estadísticas & $* *$ & $* *$ \\
\hline Coeficiente de variación $\%$ & 9.80 & 11.63 \\
\hline
\end{tabular}

(*)Promedios con la misma letra no difieren estadísticamente según prueba de Duncan al 5\% de significancia

$(\mathrm{FQ})=$ Fertilización química .

\section{Días a floración}

En el Cuadro 2, se observan los promedios de los días a floración registrados durante el desarrollo del cultivo. Los valores realizados al análisis de varianza alcanzaron significancia estadística al $5 \%$ de probabilidad.

En la evaluación realizada se encontró que el mayor número de días se encontró en los tratamientos: Humus $3000 \mathrm{Kg} / \mathrm{ha} \mathrm{(57.25} \mathrm{días),} \mathrm{Bocashi}$ $3500 \mathrm{Kg} / \mathrm{ha}$ (57.5 días), Bocashi $4000 \mathrm{Kg} / \mathrm{ha} \mathrm{(57.25} \mathrm{días),} \mathrm{Fossil} 100 \mathrm{Kg} / \mathrm{ha}$ (58 días), Algasoil (200 Kg/ha) y FQ (57.75 días), los cuales fueron estadísticamente iguales entre sí. el menor valor se registró en el tratamiento Humus (54.5 días). El coeficiente de variación fue 3.21\%.

\section{Días a maduración}

En el Cuadro 2, se observaron los promedios de números de días a maduración de frutos evaluados en los tratamientos estudiados. Los valores alcanzaron alta significancia estadística al $5 \%$ de probabilidades.

Se encontró que el mayor número de días a maduración se presentó en el tratamiento Testigo (98.75 días), el cual fue estadísticamente superior a los demás tratamientos. El menor valor se registró en los tratamientos: Humus $3000 \mathrm{Kg} / \mathrm{ha}$ ), Humus $4000 \mathrm{Kg} / \mathrm{ha}$, Bocashi $3500 \mathrm{Kg} / \mathrm{ha}$, Bocashi $4000 \mathrm{Kg} / \mathrm{ha}$, Fossil $100 \mathrm{Kg} / \mathrm{ha}$, Fossil $150 \mathrm{Kg} / \mathrm{ha}$, Algasoil $200 \mathrm{Kg} / \mathrm{ha}$ y Algasoil 400 $\mathrm{Kg} / \mathrm{ha}$ y FQ, (96, 96, 95.75, 96.25, 95.5, 96.25, 95.5, 96, 95.75, $96.75 \mathrm{Y}$ 96.75 días respectivamente). El coeficiente de variación fue $1.00 \%$.

Cuadro 2. Promedios de días a floración y días a maduración del pimiento con aplicación de enmiendas orgánicas, como complemento a la fertilización química.

\begin{tabular}{|c|c|c|}
\hline \multirow{2}{*}{ Tratamientos (*) } & \multicolumn{2}{|c|}{ Altura de planta (cm) } \\
\cline { 2 - 3 } & $\begin{array}{c}\text { Días a la } \\
\text { floración }\end{array}$ & $\begin{array}{l}\text { Días a la } \\
\text { maduración }\end{array}$ \\
\hline FQ + Humus $3000 \mathrm{Kg} / \mathrm{ha}$ & 57,25 & 96 \\
\hline $\mathrm{FQ}+$ Humus $4000 \mathrm{Kg} / \mathrm{ha}$ & 54,5 & 96 \\
\hline $\mathrm{FQ}+$ Bocashi $4000 \mathrm{Kg} / \mathrm{ha}$ & 57,5 & 95,75 \\
\hline FQ + Bocashi $3500 \mathrm{Kg} / \mathrm{ha}$ & 57,25 & 96,25 \\
\hline FQ + Fossil $100 \mathrm{Kg} / \mathrm{ha}$ & 58 & 95,5 \\
\hline FQ + Fossil $150 \mathrm{Kg} / \mathrm{ha}$ & 57 & 96 \\
\hline FQ + Algasoil $200 \mathrm{Kg} / \mathrm{ha}$ & 57,25 & 96,75 \\
\hline FQ + Algasoil 400 Kg/ha & 56,75 & 96,75 \\
\hline FQ. 160-60-130-50-5 & 57,75 & 96,75 \\
\hline
\end{tabular}




\begin{tabular}{|c|c|c|}
\hline $\begin{array}{c}\text { Testigo convencional } \\
90-18-54\end{array}$ & 56,25 & 98,75 \\
\hline Promedios & $\mathbf{5 6 . 9 5}$ & $\mathbf{9 6 . 3 5}$ \\
\hline Significacncia estadísticas & $* *$ & $* *$ \\
\hline Coeficiente de variación $\%$ & 3.21 & $1 \%$ \\
\hline
\end{tabular}

(*) Todos los tratamientos llevaron programa de fertilización PQ. Promedios con la misma letra no difieren estadísticamente según prueba de Duncan al 5\% de significancia.

\section{Días a cosecha}

$(\mathrm{FQ})=$ Fertilización química.

En el Cuadro 3, se observan los promedios de días a cosecha evaluados en los tratamientos estudiados. Realizando el análisis de varianza se encontró alta significancia estadística al 5\% de probabilidades.

Se encontró que el mayor número de días se presentó en el tratamiento testigo (77.5 días), el cual fue estadísticamente igual a los tratamientos: Fossil $150 \mathrm{Kg} / \mathrm{ha}(76.75$ días) y Bocashi $4000 \mathrm{Kg} / \mathrm{ha}$ (76.5 días), pero superiores a los demás tratamientos. El menor número de días se registró en los tratamientos Humus $3000 \mathrm{Kg} / \mathrm{ha}$ (75.5 días) y Bocashi $3500 \mathrm{Kg} / \mathrm{ha}(75.5$ días). El coeficiente de variación fue de $1.11 \%$.

\section{Número de frutos por planta.}

En este mismo Cuadro 3, se observan los promedios de número de frutos por planta evaluados en los tratamientos estudiados. Los valores al análisis de varianza alcanzaron significacncia estadística al $5 \%$ de probabilidades.

El mayor número de frutos se presentó en el tratamiento Algasoil 200 $\mathrm{Kg} / \mathrm{ha}$ (35.75 frutos), el cual fue estadísticamente igual a los tratamientos: Humus $3000 \mathrm{Kg} / \mathrm{ha}$ (27 frutos), Humus $4000 \mathrm{Kg} / \mathrm{ha}$ (34.25 frutos), Bocashi $3500 \mathrm{Kg} / \mathrm{ha}$ (26.25 frutos), Bocashi $4000 \mathrm{Kg} / \mathrm{ha}$ (27.5 frutos), Fossil 100 $\mathrm{Kg} / \mathrm{ha}$ (29.25 frutos), Fossil $150 \mathrm{Kg} / \mathrm{ha}$ (33.75 frutos), Algasoil $400 \mathrm{Kg} / \mathrm{ha}$ (29.25 frutos) y PQ (28.5 frutos), pero superiores al tratamiento Testigo; el cual tuvo el menor número de frutos ( 25 frutos). El coeficiente de variación fue de $24.25 \%$.

Cuadro 3. Promedios de días a cosecha y número de frutos por planta de pimiento con aplicación de enmiendas orgánicas, como complemento a la fertilización química.

\begin{tabular}{|c|c|c|}
\hline \multirow{2}{*}{ Tratamientos (*) } & \multicolumn{2}{|c|}{ Altura de planta $(\mathbf{c m})$} \\
\cline { 2 - 3 } & $\begin{array}{c}\text { Días de } \\
\text { cosecha }\end{array}$ & $\begin{array}{c}\text { Número de } \\
\text { frutos/planta }\end{array}$ \\
\hline FQ + Humus $3000 \mathrm{Kg} / \mathrm{ha}$ & 75,5 & 27 \\
\hline $\mathrm{FQ}+$ Humus $4000 \mathrm{Kg} / \mathrm{ha}$ & 76,25 & 35,25 \\
\hline $\mathrm{FQ}+$ Bocashi $4000 \mathrm{Kg} / \mathrm{ha}$ & 75,5 & 26,25 \\
\hline $\mathrm{FQ}+$ Bocashi $3500 \mathrm{Kg} / \mathrm{ha}$ & 76,5 & 27,5 \\
\hline FQ + Fossil 100 Kg/ha & 76,25 & 29,75 \\
\hline FQ + Fossil $150 \mathrm{Kg} / \mathrm{ha}$ & 76,75 & 33,75 \\
\hline
\end{tabular}




\begin{tabular}{|c|c|c|}
\hline FQ + Algasoil 200 Kg/ha & 75,75 & 35,75 \\
\hline FQ + Algasoil 400 Kg/ha & 76 & 29,25 \\
\hline FQ. 160-60-130-50-5 & 75,75 & 28,5 \\
\hline $\begin{array}{c}\text { Testigo convencional } \\
90-18-54\end{array}$ & 77,5 & 25 \\
\hline Promedios & $\mathbf{7 6 , 1 8}$ & $\mathbf{2 9 . 7}$ \\
\hline Significacncia estadísticas & $* *$ & $* *$ \\
\hline Coeficiente de variación \% & 1.11 & 24.25 \\
\hline
\end{tabular}

(*) Todos los tratamientos llevaron programa de fertilización PQ. Promedios con la misma letra no difieren estadísticamente según prueba de Duncan al 5\% de significancia.

\section{Diámetro de frutos}

$(\mathrm{FQ})=$ Fertilización química.

En el Cuadro 4, se observa los promedios de diámetro de frutos obtenidos en los tratamientos estudiados. Los valores, al realizar el análisis de varianza, alcanzaron alta significancia estadística al $5 \%$ de probabilidades.

El mayor diámetro se encontró en el tratamiento Humus $4000 \mathrm{Kg} / \mathrm{ha}$ $(4.54 \mathrm{~cm})$, el cual fue estadísticamente igual a los demás tratamientos, con excepción del testigo $(4.09 \mathrm{~cm})$ el cual fue estadísticamente inferior; presentando el menor promedio. El coeficiente de variación fue 6 .

\section{Tamaño de frutos}

En el Cuadro 4, se muestran los promedios de tamaño de fruto encontrados en los tratamientos estudiados. Se alcanzó alta significancia estadística al 5\% de probabilidades.

El mayor tamaño se encontró en los tratamientos Bocashi $3500 \mathrm{Kg} / \mathrm{ha}$ $(11.96 \mathrm{~cm})$ y Bocashi $4000 \mathrm{Kg} / \mathrm{ha}(11.95 \mathrm{~cm})$, los cuales fueron estadísticamente iguales a los demás tratamientos. Sin embargo fueron estadísticamente superiores al tratamiento PQ que obtuvo el menor promedio $(10.86 \mathrm{~cm})$. El coeficiente de variación fue de $6.06 \%$.

Cuadro 4. Promedios diámetro de frutos y longitud de frutos de pimiento con aplicación de enmiendas orgánicas, como complemento a la fertilización química.

\begin{tabular}{|c|c|c|}
\hline \multirow{2}{*}{ Tratamientos (*) } & \multicolumn{2}{|c|}{ Altura de planta $(\mathbf{c m})$} \\
\cline { 2 - 3 } & $\begin{array}{c}\text { Días de frutos } \\
\text { (cm) }\end{array}$ & $\begin{array}{c}\text { Longitud de } \\
\text { frutos }(\mathbf{c m})\end{array}$ \\
\hline FQ + Humus $3000 \mathrm{Kg} / \mathrm{ha}$ & $4,19 \mathrm{ab}$ & $11,45 \mathrm{ab}$ \\
\hline $\mathrm{FQ}+$ Humus $4000 \mathrm{Kg} / \mathrm{ha}$ & $4,54 \mathrm{a}$ & $11,88 \mathrm{ab}$ \\
\hline $\mathrm{FQ}+$ Bocashi $4000 \mathrm{Kg} / \mathrm{ha}$ & $4,24 \mathrm{ab}$ & $11,96 \mathrm{a}$ \\
\hline $\mathrm{FQ}+$ Bocashi $3500 \mathrm{Kg} / \mathrm{ha}$ & $4,26 \mathrm{ab}$ & $11,95 \mathrm{a}$ \\
\hline FQ + Fossil $100 \mathrm{Kg} / \mathrm{ha}$ & $4,35 \mathrm{ab}$ & $11,56 \mathrm{ab}$ \\
\hline FQ + Fossil $150 \mathrm{Kg} / \mathrm{ha}$ & $4,45 \mathrm{ab}$ & $11,81 \mathrm{ab}$ \\
\hline FQ + Algasoil $200 \mathrm{Kg} / \mathrm{ha}$ & $4,24 \mathrm{ab}$ & $11,72 \mathrm{ab}$ \\
\hline FQ + Algasoil 400 Kg/ha & $4,52 \mathrm{ab}$ & $11,66 \mathrm{ab}$ \\
\hline FQ. 160-60-130-50-5 & $4,44 \mathrm{ab}$ & $10,86 \mathrm{~b}$ \\
\hline
\end{tabular}




\begin{tabular}{|c|c|c|}
$\begin{array}{c}\text { Testigo convencional } \\
90-18-54\end{array}$ & $4,09 \mathrm{~b}$ & $11,48 \mathrm{ab}$ \\
\hline Promedios & $\mathbf{4 . 3 3}$ & $\mathbf{1 1 . 6 3}$ \\
\hline Significacncia estadísticas & $* *$ & $* *$ \\
\hline Coeficiente de variación $\%$ & 6.99 & 6.05 \\
\hline
\end{tabular}

(*) Todos los tratamientos llevaron programa de fertilización PQ. Promedios con la misma letra no difieren estadísticamente según prueba de Duncan al 5\% de significancia.

\section{Peso de frutos}

$(\mathrm{FQ})=$ Fertilización química

En el Cuadro 5, se pueden observar los promedios de peso de frutos de los tratamientos estudiados. Los valores, del análisis de varianza, alcanzó alta significancia estadística al 5\% de probabilidades.

El mayor peso se encontró en el tratamiento Algasoil $200 \mathrm{Kh} / \mathrm{ha}$ con 125.59 gramos, el cual fue estadísticamente igual a los tratamientos: Humus $4000 \mathrm{Kg} / \mathrm{ha}$ (115.09 gramos), Bocaschi $3500 \mathrm{Kg} / \mathrm{ha}$ (118.87 gramos). Fossil $100 \mathrm{Kg} / \mathrm{ha}$ (88.57 gramos), Fossil $150 \mathrm{Kg} / \mathrm{ha}$ (112.52 gramos), Algasoil 400 $\mathrm{Kg} / \mathrm{ha}$ (102.13 gramos) y PQ (99.29 gramos), pero superior al testigo que obtuvo el menor promedio (72.14 gramos). El coeficiente de variación fue de $26.86 \%$.

\section{Rendimiento por hectárea}

En el Cuadro 5, se registran los promedios del rendimiento por hectárea de los tratamientos. Los valores, realizados por el análisis de varianza alcanzaron alta significancia etadísitica al 5\% de probabilidades.

El mayor rendimiento se encontró en el tratamiento Algasoil $200 \mathrm{Kg} / \mathrm{ha}$ con $59.88 \mathrm{t} / \mathrm{ha}$, el cual fue estadísticamente igual al tratamiento Humus 4000 $\mathrm{Kg} / \mathrm{ha}(52.31 \mathrm{Kg} / \mathrm{ha})$, pero superior a los demás tratamientos. El menor promedio se encontró en el testigo con 23.85 t/ha. Para este caso el coeficiente de variación fue de $30.95 \%$.

Cuadro 5. Promedios peso de frutos y rendimiento de pimiento por hectárea con aplicación de enmiendas orgánicas, como complemento a la fertilización química.

\begin{tabular}{|c|c|c|}
\hline \multirow{2}{*}{ Tratamientos (*) } & \multicolumn{2}{|c|}{ Altura de planta (cm) } \\
\cline { 2 - 3 } & Peso de fruto $(\mathbf{g})$ & $\begin{array}{c}\text { Rendimiento } \\
\text { Ton/ha }\end{array}$ \\
\hline FQ + Humus $3000 \mathrm{Kg} / \mathrm{ha}$ & $84,86 \mathrm{bc}$ & $30,20 \mathrm{~cd}$ \\
\hline FQ + Humus $4000 \mathrm{Kg} / \mathrm{ha}$ & $115,09 \mathrm{ab}$ & $52,31 \mathrm{ab}$ \\
\hline FQ + Bocashi $4000 \mathrm{Kg} / \mathrm{ha}$ & $118,87 \mathrm{ab}$ & $41,60 \mathrm{bc}$ \\
\hline FQ + Bocashi $3500 \mathrm{Kg} / \mathrm{ha}$ & $82,34 \mathrm{bc}$ & $31,54 \mathrm{~cd}$ \\
\hline FQ + Fossil $100 \mathrm{Kg} / \mathrm{ha}$ & $88,57 \mathrm{abc}$ & $35,19 \mathrm{bcd}$ \\
\hline FQ + Fossil $150 \mathrm{Kg} / \mathrm{ha}$ & $112,52 \mathrm{ab}$ & $48,99 \mathrm{bcd}$ \\
\hline FQ + Algasoil $200 \mathrm{Kg} / \mathrm{ha}$ & $125,52 \mathrm{ab}$ & $59,88 \mathrm{a}$ \\
\hline FQ + Algasoil $400 \mathrm{Kg} / \mathrm{ha}$ & $125,59 \mathrm{a}$ & $40,14 \mathrm{bcd}$ \\
\hline FQ. 160-60-130-50-5 & $102,13 \mathrm{abc}$ & $37,80 \mathrm{bcd}$ \\
\hline Testigo convencional & $99,29 \mathrm{abc}$ & $23,85 \mathrm{~d}$ \\
\hline
\end{tabular}




\begin{tabular}{|c|c|c|}
\hline $90-18-54$ & & \\
\hline Promedios & $\mathbf{1 0 0 . 1 4}$ & $\mathbf{4 0 . 1 5}$ \\
\hline Significacncia estadísticas & $* *$ & $* *$ \\
\hline Coeficiente de variación $\%$ & 26.86 & 30.95 \\
\hline
\end{tabular}

(*) Todos los tratamientos llevaron programa de fertilización PQ. Promedios con la misma letra no difieren estadísticamente según prueba de Duncan al 5\% de significancia.

\section{DISCUSIÓN}

$(\mathrm{FQ})=$ Fertilización química.

Basados en los resultados del presente ensayo se demostró, que la aplicación de materia orgánica en combinación de un programa de fertilización, incidieron en el comportamiento agronómico del cultivo de pimiento, bajo las condiciones de campo en que se desarrolló el mismo.

Como consecuencia de las aplicaciones de fertilizantes y enmiendas realizadas, se encontró que las mismas tuvieron influencia sobre la altura de la planta en las mediciones realizadas en los tratamientos evaluados pues la aplicación de fertilización química más abono orgánico influye sobre el comportamiento morfológico del cultivo.

Realizados los análisis de estadística también se puede mencionar que el material Híbrido Salvador utilizado en este ensayo, presenta una prolongación en los días correspondientes a la floración y maduración, bajo las aplicaciones de materia orgánica funcional, factor que sumado a la aplicación de fertilización química incide en el rendimiento. Esto se explica por las apreciaciones de Ecuaquímica (2008), quienes manifiestan que Algasoil es un acondicionador de suelo, promueve la generación de fitoalexinas y sus metabólicos, las mismas que pertenecen a un nuevo grupo de sustancias que protegen al cultivo del ataque de enfermedades.

Es importante recalcar que no se apreció una alta incidencia de plagas, aunque la presencia de Fusarium se presentó en los tratamientos que recibieron aplicaciones de Bocashi en diversas dosis. En razón de que el Bocashi resulta de la fermentación de desechos de carácter vegetal y microorganismos para activar el proceso fermentativo, posee una alta carga de microorganismos benéficos y en ciertos casos patógenos según su proceso de descomposición.

El mejor comportamiento agronómico del fruto se encontró en el tratamiento Bocashi $4000 \mathrm{Kg} / \mathrm{ha}$, el cual presentó mejores características en número de frutos, diámetro de frutos y longitud de frutos, siendo este estadísticamente igual a la dosis de Bocashi $3500 \mathrm{Kg} / \mathrm{ha}$. Los abonos orgánicos fermentados del tipo "Bocashi"son ricos en nutrientes para la planta e incorporan gran cantidad de microorganismos benéficos, resultado de un proceso de fermentación con un grado prácticamente nulo de descomposición. Es un tipo de abono fermentado, que utilizado para la fertilización de las hortalizas, reduce los costos de producción. 
Los rendimientos presentados sobrepasaron el promedio obtenido a nivel nacional, bajo las condiciones del ensayo. El mayor efecto se presentó en el tratamiento Algasoil 200Kg (58.88) que están por encima del testigo.

\section{CONCLUSIONES Y RECOMENDACIONES}

En la presente investigación se interpretó los resultados y con ellos se realizó las siguientes conclusiones y recomendaciones.

1. En altura de planta se registró mayores promedios en esta variable con los tratamientos FQ + Humus $4000 \mathrm{Kg} / \mathrm{ha}$ (30.75), y FQ + Humus $3000 \mathrm{Kg} / \mathrm{ha}(27.2 \mathrm{~cm})$.

2. En lo referente a días de floración se obtuvo mayor número de días con FQ + Fossil (58 días) y menor número con FQ + Humus 3000 $\mathrm{Kg} / \mathrm{ha}$ (57.5 días) FQ + Bocashi $4000 \mathrm{Kg} / \mathrm{ha} \mathrm{(57.25} \mathrm{días)} \mathrm{y} \mathrm{FQ} \mathrm{+}$ Fossil $100 \mathrm{Kg} / \mathrm{ha}$ (58 días)

3. El mayor diámetro de fruto se dio con aplicación de $\mathrm{FQ}+$ Humus $4000 \mathrm{Kg} / \mathrm{ha}(4.54 \mathrm{~cm})$

4. Mayor longitud de frutos se obtuvo con $\mathrm{FQ}+$ Bocashi $3500 \mathrm{Kg} / \mathrm{ha}$ $(11.96 \mathrm{~cm})$

5. El tratamiento $\mathrm{FQ}+$ Algasoil $200 \mathrm{Kg} / \mathrm{ha}$ (35.75 frutos) obtuvo mayor número de fruto, siendo el menor promedio el tratamiento testigo ( 25 frutos/planta).

6. Se encontró significancia estadística en el peso de fruto siendo el tratamiento FQ + Algasoil $200 \mathrm{Kg} / \mathrm{ha}$ con 125.59 gramos el de mayor peso.

7. En consecuencia a días de maduración se observó que el mayor número de días a maduración se presentó en el tratamiento testigo convencional (98.75 días)

8. El mayor rendimiento se encontró en el tratamiento Algasoil 200 $\mathrm{Kg} / \mathrm{ha}$ con $59.88 \mathrm{~T} / \mathrm{ha}$ y el menor promedio se encontró en el Testigo convencional $23.85 \mathrm{~T} / \mathrm{ha}$.

Luego de las conclusiones y análisis realizados se recomienda:

9. Realizar las aplicaciones de enmiendas orgánicas como complemento a la fertilización química.

10. Aplicar Algasoil en dosis de $200 \mathrm{Kg} / \mathrm{ha}$, como complemento a la fertilización química para aumentar el rendimiento del cultivo de pimiento.

11. Realizar trabajos adicionales con nuevas fuentes y materiales de siembra. 


\section{References:}

1. CIAT (2003). El cultivo de hortalizas en Colombia. Ministerio de agricultura y territorio. Bogotá. 39 p.

2. Colacelli, N. A. (1997). SUELOS: EL HUMUS DE LOMBRIS. Producción,1. Consultado el 10 de Junio del 2016: Información disponible en http://www.produccion.com.ar/1997/indice19.htm.

3. ECUAQUÍMICA, EC. (2008). Algasoil: fertilizante orgánico granulado de algas marinas. (en línea) Guayaquil, ec. Consultado 8 de Mayo 2016. Disponible en http://www.ecuaquímica.com

4. Espinoza, J. (2007). Manual técnico de fertilización. Institute of Investigation of Phosphorus. Rio de Janeiro-Brasil p. 42.

5. Javier de Grazia, P.A. (2006). Efectos de sustratos con compost y fertilización nitrogenada sobre la fotosíntesis, precocidad y rendimiento de pimiento (Capsicum annuum). Scielo. Consultado el 21 de Junio del 2016. Disponible en: http://www.scielo.cl/scielo.php?pid=S0718$16202007000300003 \&$ script $=$ sci arttext.

6. Revisado en: www.mundoverde2015

7. SUQUILANDA M. (2014). Producción orgánica de hortalizas en la sierra norte y central del ecuador. p 240. 